\title{
Can pregnancy-associated plasma protein-A be a marker for the assessment of atherosclerosis risk in patients with chronic plaque psoriasis?
}

\author{
Mualla Polat ${ }^{1}$, Guler Bugdayci², Asli Sahin ${ }^{3}$, Hatice Kaya ${ }^{1}$, Tuna Sezer ${ }^{3}$, Serkan Ozturk \\ ${ }^{1}$ Department of Dermatology, Faculty of Medicine, Abant Izzet Baysal University, Bolu, Turkey \\ ${ }^{2}$ Department of Biochemistry, Faculty of Medicine, Abant Izzet Baysal University, Bolu, Turkey \\ ${ }^{3}$ Department of Dermatology, Izzet Baysal Education and Research Hospital, Bolu, Turkey \\ ${ }^{4}$ Department of Cardiology, Faculty of Medicine, Abant Izzet Baysal University, Bolu, Turkey
}

Adv Dermatol Allergol 2016; XXXIII (5): 340-344

DOI: $10.5114 / a d a .2016 .62839$

\begin{abstract}
Introduction: Psoriasis is an immune-mediated chronic inflammatory dermatosis. Several studies have shown that patients with psoriasis have a much greater risk of cardiovascular diseases than the normal population. The chronic inflammation observed in psoriasis is thought to have a role in the development of atherosclerosis and vascular endothelial injury.

Aim: To examine serum pregnancy-associated plasma protein-A (PAPP-A) levels, which has been regarded as a marker of early stage atherosclerosis in patients with psoriasis that do not have concurrent conventional cardiovascular risk markers.

Material and methods: Forty-one patients diagnosed with a chronic plaque type of psoriasis and 42 equally matched healthy volunteers were included in this study. The PAPP-A levels were compared between patient and control groups and the association between PAPP-A levels and disease duration and severity were evaluated in the patient group.

Results: Statistically, serum PAPP-A levels were significantly higher in the psoriasis group than in the control group $(p=0.015)$. Serum PAPP-A levels were found to be positively correlated with severity $(p=0.036, r=0.329)$ and duration ( $p=0.014, r=0.269$ ) of the disease.

Conclusions: As a marker of early stage atherosclerosis, PAPP-A levels were elevated in the psoriasis group and were correlated with disease duration and severity. This elevation reveals the presence of atherosclerosis in patients with psoriasis. Further studies are needed to confirm the use of PAPP-A as an available and inexpensive screening test and cardiovascular risk assessment for all centers.
\end{abstract}

Key words: psoriasis, pregnancy associated plasma protein-A, atherosclerosis.

\section{Introduction}

Psoriasis is a chronic recurrent inflammatory skin disease involving the knee, elbow, hairy skin, sacral area, and the umbilical surrounding and nails. It is characterized by sharp-edged, erythematous, silvery squamous papules, and plaques [1]. Recent studies have shown that patients who are of a young age with severe plaque type psoriasis will have a two-fold greater risk of cardiovascular disease and mortality compared to the normal population [2-5]. Apart from known risks such as hypertension, diabetes mellitus, smoking and alcohol intake, it is thought that the chronic inflammation observed in psoriasis also has a major role in the development of atherosclerosis and vascular endothelial injury [1-7]. Immunological factors mediated by Th1, Th17 activation, and reduced $T$ regulatory (Treg) cell function, and known to be important in pathogenesis of chronic inflammatory diseases such as psoriasis and psoriatic arthritis, have also been shown to play a role in the pathogenesis of atherosclerosis and cardiovascular diseases [8-10].

Pregnancy-associated plasma protein-A (PAPP-A) is a heterotetramerer protein complex released from the placenta in pregnancy; it is a high molecular weight metalloproteinase made up of two subunits linked through

Address for correspondence: Mualla Polat MD, Department of Dermatology, Faculty of Medicine, Abant Izzet Baysal University, Golkoy, Bolu, Turkey, phone: +90 37425346 56, fax: +90 37425346 15, e-mail: polatmualla@gmail.com Received: 1.09.2015, accepted: 2.10.2015. 
a disulfide bridge [11]. The PAPP-A has been shown to be effective on insulin-like growth factor (IGF-1), which plays an important role in atherosclerosis pathogenesis. The PAPP-A causes an increase in the free form of IGF-1 through accelerated degradation of insulin like growth factor binding protein 4 (IGFBP4). Upon this process, it expedites atherosclerosis development indirectly and stands as an important risk factor for unstable angina and acute coronary syndromes $[12,13]$. Studies have shown that PAPP-A concentrations are elevated in unstable atherosclerotic plaques, and serum levels are much higher in cases with acute coronary syndrome than in the normal population [14-16]. It is currently thought that it can be used as a non-invasive marker for detection of early stage atherosclerosis $[17,18]$.

\section{Aim}

In our study, serum PAPP-A levels were measured in patients with psoriasis that did not have concurrent known cardiovascular risk factors and an equally matched control group. By examining the correlation of PAPP-A levels with disease severity and duration, we aimed to determine the independent risk factor for the association of psoriasis and cardiovascular disease, and whether serum PAPP-A levels can be used as a marker of this risk.

\section{Material and methods}

This study was conducted on a control group of healthy volunteers and patients diagnosed with chronic plaque type psoriasis who came to the Abant Izzet Baysal University Faculty of Medicine Dermatology Department. Two patients were excluded from the study due to the development of pustular lesions, and the study was performed on 41 patients and a group of 42 equally matched controls. The study was approved by the Bolu Clinical and Laboratory Research Ethical Committee; decision number 2014/99-188. Patients included in the study were: (i) between ages of 18-55 years; (ii) diagnosed with chronic plaque type psoriasis who were receiving only topical treatment or phototherapy; and (iii) stopped systemic treatment at least 1 month prior to the study. Additionally, the following was accepted as exclusion criteria for selection of both patient and control groups: history of present or previous myocardial infarction, stroke, peripheral arterial disease, coronary heart disease, angina pectoris, cardiac failure, left ventricular ejection fraction (LVEF) $<50 \%$, atrial fibrillation, ischemic echocardiographic changes, presence of a cardiac pacemaker, prolonged QRS time ( $\geq 120 \mathrm{~ms}$ ), hypertrophic cardiomyopathy, valvular heart disease, congenital heart disease, diabetes mellitus, obesity, uncontrolled hypertension (blood pressure at rest $\geq 140 / 90 \mathrm{~mm} \mathrm{Hg}$ ), hepatic and renal dysfunction (creatinine $>1.5 \mathrm{mg} / \mathrm{dl}$, aspartate aminotransferase level above twice the upper limit), history of malignancy, local or systemic infection, disturbance of the thyroid function, other systemic inflammatory diseases and/or psoriatic arthritis, and previous use of biological agents such as tumor necrosis factor $\alpha$ (TNF- $\alpha$ ) blocker.

Questions about age, sex, disease duration, presence of another family member with psoriasis, previously received treatments, and the current treatment were asked and recorded in patient forms. Systemic and dermatological examinations were performed for each patient and body mass index (BMI), psoriasis area, and severity index (PASI) were calculated. Blood pressure was measured and blood samples were obtained following a 12hour fasting period.

Body mass index was calculated by body weight in kilograms divided by the square of the body height in meters, and was universally expressed in $\mathrm{kg} / \mathrm{m}^{2}$. Insulin resistance was related with the tendency for some morbidities such as obesity, type 2 diabetes, hypertension, dyslipidemia, coronary artery disease, and is associated with the amount of body fat tissue. For this reason, our study comprised individuals who had BMI within the normal limits $\left(19-25 \mathrm{~kg} / \mathrm{m}^{2}\right)$.

The PASI is one of the frequently used scales for determination of the severity of psoriasis. It is used to grade disease findings such as erythema, desquamation, and induration at four body areas according to their anatomical localizations. The PASI scores vary between 0 and 72, and the score numerically increases in parallel with the severity of the disease.

The control group consisted of healthy volunteers, and attention was paid to create no difference between patient and control groups with regards to age, sex, and BMI.

\section{Collection and analysis of blood samples}

Venous blood samples were obtained following $12 \mathrm{~h}$ of fasting from all participants at 9 a.m. in a sitting position. Blood samples were withdrawn into sample tubes containing a gel separator with clot activator (Vacuette ${ }^{\circledR}$, Greiner, Bio-one GmbH, Frickenhausen, Germany). Upon withdrawal, sample tubes with a gel separator were held for $30 \mathrm{~min}$ and then centrifuged at 1,250 g for $15 \mathrm{~min}$. Obtained serum samples were stored at $-80^{\circ} \mathrm{C}$. Other blood samples that were taken into vacuum tubes were analyzed on the same day with samples that were sent to the appropriate unit of the laboratory.

Serum glucose, triglyceride, and high-density lipoproteins (HDL)-cholesterol levels were analyzed with an Abbott commercial assay kit on Abbott C8000 Architect (Abbott Laboratories, Abbott Park, IL, USA) biochemistry autoanalyzer. Low-density lipoprotein (LDL)-cholesterol was calculated according to Friedewald formula (total cholesterol - (HDL-cholesterol + VLDL-cholesterol); VLDL- 
cholesterol was calculated using the formula: triglyceride/5.

Serum PAPP-A levels were analyzed with a Roche commercial assay kit on Cobas ${ }^{\circledR}$ e601 (Roche Diagnostics, Mannheim, Germany) immune-analyzer using enzyme chemiluminescence immunoassay technique. The serum PAPP-A measurement range with Roche commercial assay kit was 4-10,000 mIU/l. The intra-assay and inter-assay variation coefficient was below $2.0 \%$ in two different analyte concentrations of 52 and $346 \mathrm{mIU} / \mathrm{I}$. Serum PAPP-A levels in patient and control groups are given as two different categorical variables as detectable $(<4 \mathrm{mlU} / \mathrm{ml})$ and non-detectable (> $4 \mathrm{mlU} / \mathrm{ml})$.

\section{Statistical analysis}

Statistical analysis was performed with SPSS (Statistical Package for Social Sciences) for Windows 22.0 package software. Normality assessment was made with Kolmogorov-Smirnov test. Continuous variables were expressed as mean \pm standard deviation; categorical variables were expressed as \% (percentage) values. In order to examine the difference between two groups for variables that meet a normal distribution assumption, a t-test for independent samples was used. For variables that do not fit into normal distribution, the difference between two independent groups was analyzed with Mann-Whitney $U$ test as a non-parametric test. Differences between groups for categorical variables were analyzed with the $\chi^{2}$ test. For correlation analysis of normally distributed variables, a Pearson correlation test was used, whereas correlation analysis was made with the Spearman correlation test for non-normally distributed variables. A statistical significance level was accepted as $\alpha=0.05$ for all tests; for $p$-values below this level ( $p<$
0.05), null hypothesis was denied and it was stated that there is a statistically significant difference.

\section{Results}

The study included totally 83 participants, consisting of 41 patients diagnosed with chronic plaque type psoriasis who did not have cardiovascular risk factors and 42 healthy individuals. There were 21 (51\%) males and 20 (49\%) females in the patient group. There were 22 (52\%) females and 20 (52\%) males in the control group. The distribution of age, sex, disease duration, PASI, BMI, serum glucose, lipid levels in patient and control groups is given in Table 1 with corresponding $p$-values.

Serum PAPP-A levels were greater than $4 \mathrm{mIU} / \mathrm{ml}$ in 21 (51\%) patients and less than $4 \mathrm{mIU} / \mathrm{ml}$ in 20 (49\%) patients with psoriasis; whereas in the control group, $9(21 \%)$ individuals had serum PAPP-A levels greater than $4 \mathrm{mIU} / \mathrm{ml}$ and 33 (79\%) individuals had serum PAPP-A levels less than $4 \mathrm{mIU} / \mathrm{ml}$. There was a statistical difference between patient and control groups with $\chi^{2}$ test $(p=0.015)$ (Table 2). The PAPP-A levels in patient and control groups are shown in Figure 1.

In 17 of the 21 male patients diagnosed with psoriasis, serum PAPP-A levels were greater than $4 \mathrm{mIU} / \mathrm{ml}$, and in 4 male patients, serum PAPP-A levels were less than $4 \mathrm{mIU} / \mathrm{ml}$. In 7 of the 20 female patients, serum PAPPA levels were greater than $4 \mathrm{mIU} / \mathrm{ml}$, and in 13 female patients serum PAPP-A levels were less than $4 \mathrm{mIU} /$ $\mathrm{ml}$. Within the patient group, there was a statistically significant difference between male and female patients regarding serum PAPP-A levels ( $p=0.014)$. There were correlations between the serum PAPPA level and PASI score $(r=0.329, p=0.036)$ and be-

Table 1. Clinical and laboratory findings in patients with psoriasis and control subjects

\begin{tabular}{|c|c|c|c|}
\hline Parameter & $\begin{array}{c}\text { Patients }(N=41) \\
\text { Mean } \pm \text { SD }\end{array}$ & $\begin{array}{c}\text { Controls }(N=42) \\
\text { Mean } \pm \text { SD }\end{array}$ & $P$-value \\
\hline Age [years] & $36.76 \pm 9.61$ & $34.26 \pm 7.83$ & 0.198 \\
\hline Gender (F/M) & $20 / 21$ & $22 / 20$ & 0.745 \\
\hline Duration of psoriasis [months] & $174.88 \pm 91.1$ & & \\
\hline PASI & $5 \pm 1.29$ & & \\
\hline $\mathrm{BMI}\left[\mathrm{kg} / \mathrm{m}^{2}\right]$ & $23.00 \pm 1.93$ & $23.28 \pm 1.91$ & 0.515 \\
\hline Glucose [mg/dl] & $88.15 \pm 6.99$ & $88.88 \pm 8.09$ & 0.660 \\
\hline Cholesterol [mg/dl] & $172.92 \pm 30.80$ & $178.61 \pm 26.53$ & 0.369 \\
\hline Triglyceride [mg/dl] & $109.07 \pm 40.64$ & $97.24 \pm 45.83$ & 0.217 \\
\hline HDL-cholesterol [mg/dl] & $44.21 \pm 9.91$ & $45.29 \pm 10.49$ & 0.631 \\
\hline LDL-cholesterol [mg/dl] & $113.00 \pm 34.48$ & $120.29 \pm 29.27$ & 0.305 \\
\hline VLDL-cholesterol [mg/dl] & $22.53 \pm 10.10$ & $20.67 \pm 11.38$ & 0.233 \\
\hline
\end{tabular}

$S D$ - standard deviation, BMI - body mass index, $L D L$ - low-density lipoprotein, $H D L$ - high-density lipoprotein, VLDL - very-low-density lipoprotein. 
Table 2. Comparison of the serum PAPP-A level in study groups

\begin{tabular}{lccc}
\hline Group & $\begin{array}{c}\text { PAPP-A } \\
<4 \mathrm{mlU} / \mathrm{ml}\end{array}$ & $\begin{array}{c}\text { PAPP-A } \\
>4 \mathrm{mlU} / \mathrm{ml}\end{array}$ & $P$-value \\
\cline { 1 - 3 } Control $(n=42)$ & $33(79 \%)$ & $9(21 \%)$ & \multirow{2}{*}{0.015} \\
\cline { 1 - 3 } Psoriasis $(n=41)$ & $20(49 \%)$ & $21(51 \%)$ & \\
\hline
\end{tabular}

tween the serum PAPP-A level and disease duration $(r=0.269$ and $p=0.014)$. In patients with psoriasis, there was no correlation with other measured biochemical parameters. On the other hand, disease duration was found to be correlated with the patient age $(r=0.524, p=0.000)$, serum total cholesterol level $(r=$ 0.309, $p=0.050)$ and VLDL-cholesterol level $(r=0.330$, $p=0.035)$ according to Pearson correlation analysis, and the correlations were statistically significant.

\section{Discussion}

Psoriasis is thought to be a multifactorial, multigenetic disease mediated by T-cells. Recent studies suggest that psoriasis is not confined to the skin only, but is a systemic disease causing various comorbidities [2-5]. Compared to the general population, patients with psoriasis have an increased prevalence of cardiovascular diseases (stroke, atherosclerosis, myocardial infarction, coronary artery disease and endothelial dysfunction) regardless of the cardiovascular risk factors (hypertension, dyslipidemia, type 2 diabetes, metabolic syndrome, obesity) [2-5]. This condition has been attributed to similar immunological pathogenesis both in atherosclerosis and psoriasis [2]. Cells presenting antigens to lymph nodes lead to an increase in lymphocyte function-associated protein 1 (LFA-1) expression by activating naive T cells. Activated T cells migrate into blood vessels and adhere to endothelial cells (adhere to macrophages in atherosclerosis). T cells extravasate through the LFA-1 and intercellular adhesion molecule 1 (ICAM1) mediated process. They interact with dendritic cells, macrophages, and keratinocytes in psoriasis, while this interaction involves smooth muscle cells in atherosclerosis. In both conditions, released chemokines and cytokines initiate inflammation [19]. This idea has been supported by demonstration of CD4 T lymphocyte, monocyte/macrophage, and dendritic cell infiltration both in psoriasis and atherosclerosis plaques. In addition, the release of Th1 and Th17 type cytokines such as interferon (IFN) $-\gamma$, interleukin (IL)-2 and TNF- $\alpha$ in both conditions has been demonstrated $[2,8,19]$. Additionally, vascular endothelial growth factor (VEGF), which is a potent proangiogenic factor intensively released during psoriasis and atherosclerosis, has also been reported to play an effective role in atherosclerosis development by inducing intimal hyperplasia in endothelial cells through mitogenic effects [8-10]. Furthermore, quality of life in patients with psoriasis has been affected

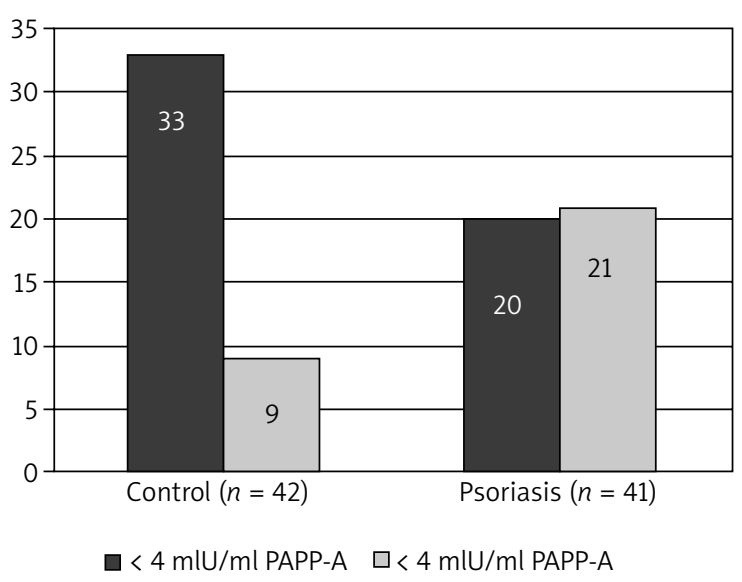

Figure 1. PAPP-A levels in the patient and control groups; detectable $(>4 \mathrm{mlU} / \mathrm{ml})$ and undetectable $(<4 \mathrm{mlU} / \mathrm{ml})$

both psychologically and physiologically, and behavioral disturbances such as tendency to smoking and alcohol consumption, and obesity may be frequently observed in these patients and present a risk of atherosclerosis [10, 20]. Thus, there is a need for a screening method to detect cardiac involvement and atherosclerosis that can develop for various reasons in patients with psoriasis [21].

The PAPP-A is a metalloproteinase that was first shown in 1974 to be released from the placenta of pregnant women [22]. However, later studies have shown that it can also be detected during wound healing in the testis, kidneys, colon, vascular smooth muscle cells and skin. The PAPP-A was shown to be effective on IGF-1, an important factor in the pathogenesis of atherosclerosis $[13,15,22]$. In 2001, Bayes-Genis et al. mentioned the role of PAPP-A in atherosclerosis development by demonstrating elevated PAPP-A levels in patients with acute coronary syndrome [15]. Later studies focused on the effect of PAPP-A on pathophysiology of atherosclerosis [2325]. Aso et al. reported a significant correlation between blood PAPP-A levels and a cardiovascular disease risk in type 2 diabetes patients and they confirmed this association with carotid intima media thickness. They stated that PAPP-A could be used as a non-invasive marker to detect early stage atherosclerosis [18].

In this study, we examined PAPP-A levels in patients with psoriasis as a possible marker for early stage atherosclerosis. In addition, we investigated whether it would be meaningful to use this common and quite inexpensive marker as a screening test for detection of comorbidities. In order to do this, we comprised equally matched patient and control groups after excluding any kind of factors that would increase a cardiovascular disease risk. According to our results, serum PAPP-A levels were significantly higher in the patient group than in the control group. There was no difference between these two groups for parameters that are known to create a predisposition to atherosclerosis such as glucose, 
BMI, and blood lipid levels. Therefore, we find that this difference in PAPP-A levels to be quite meaningful for establishing psoriasis as an independent risk factor and predisposition to atherosclerosis. Furthermore, severity and duration of the disease were found to be correlated with serum PAPP-A levels. In addition, elevated serum PAPP-A levels were statistically more frequent in male patients than female patients. These results show that atherosclerosis indicated by PAPP-A levels is more likely to develop in male patients with prolonged and severe psoriatic disease.

\section{Conclusions}

Patients with psoriasis are more prone to cardiovascular disease because of both the disease's comorbidity and patients' life-style. There is a need for a practical screening marker in order to detect the disease earlier. This study showed significant results to suggest PAPP-A as a screening tool. The PAPP-A can be an inexpensive and basic marker for detecting early atherosclerosis as a cardiovascular risk in psoriasis patients with minimal financial and workforce losses. This is the first study to investigate the association between PAPP-A and psoriasis. Further studies with a greater number of patients are needed.

\section{Conflict of interest}

The authors declare no conflict of interest.

\section{References}

1. Griffiths CEM, Baker JWN. Pathogenesis and clinical features of psoriasis. Lancet 2007; 370: 263-71.

2. El-Mongy S, Fathy H, Abdelaziz A, et al. Subclinical atherosclerosis in patients with chronic psoriasis: a potential association. J Eur Acad Dermatol Venereol 2010; 24: 661-6.

3. Sanchez-Carazo JL, López-Estebaranz JL, Guisado C. Comorbidities and health-related quality of life in Spanish patients with moderate to severe psoriasis: a cross-sectional study (Arizona study). J Dermatol 2014; 41: 673-8.

4. Gelfand JM, Neimann AL, Shin DB, et al. Risk of myocardial infarction in patients with psoriasis. JAMA 2006; 296: 1735-41.

5. Kremers HM, McEvoy MT, Dann FJ, et al. Heart disease in psoriasis. J Am Acad Dermatol 2007; 57: 347-54.

6. Owczarczyk-Saczonek AB, Nowicki R. The association between smoking and the prevalence of metabolic syndrome and its components in patients with psoriasis aged 30 to 49 years. Postep Derm Alergol 2015; 32: 331-6.

7. Sürücü HA, Aksoy N, Ozgöztas O, et al. Prolidase activity in chronic plaque psoriasis patients. Postep Derm Alergol 2015; 32: 82-7.

8. Ghazizadeh R, Shimizu H, Tosa M, Ghazizadeh M. Pathogenic mechanisms shared between psoriasis and cardiovascular disease. Int J Med Sci 2010; 7: 284-9.

9. Wu JJ, Poon KY. Association of gender, tumor necrosis factor inhibitor therapy, and myocardial infarction risk in patients with psoriasis. J Am Acad Dermatol 2013; 69: 650-1.
10. Pirro M, Stingeni L, Vaudo G, et al. Systemic inflammation and imbalance between endothelial injury and repair in patients with psoriasis are associated with preclinical atherosclerosis. Eur J Prev Cardiol 2015; 22: 1027-35.

11. Mahto S, Sharma SB, Dwivedi S, et al. Biomarkers for early detection of risk in female patients with coronary artery disease: pilot study. J Assoc Physicians India 2013; 61: 317-9.

12. Sivanandam AS, Mohan S, Kita H, et al. Studies on regulation of IGF (insulin-like growth factor)-binding protein (IGFBP) 4 proteolysis by pregnancy-associated plasma protein-A (PAPP-A) in cells treated with phorbol ester. Biochem J 2004; 379: 57-64.

13. Chen BK, Leiferman KM, Pittelkow MR, et al. Localization and regulation of pregnancy-associated plasma protein a expression in healing human skin. J Clin Endocrinol Metab 2003; 88: 4465-71.

14. Kalousová M, Zima T, Krane V, et al. Pregnancy-associated plasma protein A associates with cardiovascular events in diabetic hemodialysis patients. Atherosclerosis 2014; 236 : 263-9.

15. Bayes-Genis A, Conover CA, Overgaard MT, et al. Pregnancyassociated plasma protein $A$ as a marker of acute coronary syndromes. N Engl J Med 2001; 345: 1022-9.

16. Wang L, Jiang J, Du L, et al. The prognostic value of serum pregnancy-associated plasma protein A, S100 and high sensitivity C-reactive protein in acute ischemic stroke patients without heparin administration. Clin Biochem 2014; 47: 187-91.

17. Woelfle J, Roth CL, Wunsch R, Reinehr T. Pregnancy-associated plasma protein $\mathrm{A}$ in obese children: relationship to markers and risk factors of atherosclerosis and members of the IGF system. Eur J Endocrinol 2011; 165: 613-22.

18. Aso Y, Okumura K, Wakabayashi S, et al. Elevated pregnancy-associated plasma protein-a in sera from type 2 diabetic patients with hypercholesterolemia: associations with carotid atherosclerosis and toe-brachial index. J Clin Endocrinol Metab 2004; 89: 5713-7.

19. Al-Mutairi N, Al-Farag S, Al-Mutairi A, Al-Shiltawy M. Comorbidities associated with psoriasis: an experience from the Middle East. I Dermatol 2010; 37: 146-55.

20. Basra MK, Fenech R, Gatt RM, et al. The Dermatology Life Quality Index 1994-2007: a comprehensive review of validation data and clinical results. Br J Dermatol 2008; 159: 997-1035.

21. Örem C, Kazaz Z, Yaylı S, et al. Left ventricular systolic asynchrony: an important sign for cardiac involvement in plaquetype psoriasis. Int J Dermatol 2014; 53: 369-75.

22. Lin TM, Halbert SP, Kiefer DJ, et al. Characterization of four human pregnancy-associated plasma proteins. Am J Obstet Gynecol 1974; 118: 223-36.

23. Lund J, Qin QP, Ilva T, et al. Circulating pregnancy-associated plasma protein a predicts outcome in patients with acute coronary syndrome but no troponin I elevation. Circulation 2003; 108: 1924-6.

24. Iversen KK, Dalsgaard M, Teisner AS, et al. Usefulness of pregnancy-associated plasma protein A in patients with acute coronary syndrome. Am J Cardiol 2009; 104: 1465-71.

25. Heider P, Pfäffle N, Pelisek J, et al. Is serum pregnancy-associated plasma protein A really a potential marker of atherosclerotic carotid plaque stability? Eur J Vasc Endovasc Surg 2010; 39: 668-75. 